EPJ Web of Conferences 36, 00008 (2012)

DOI: $10.1051 /$ epjconf/20123600008

(C) Owned by the authors, published by EDP Sciences, 2012

\title{
In-medium hadron properties from lattice QCD
}

\author{
Heng-Tong Ding ${ }^{1, a}$ \\ ${ }^{1}$ Physics Department, Brookhaven National Laboratory, Upton, NY 11973, USA
}

\begin{abstract}
I review recent results from lattice QCD calculations on the in-medium hadron properties. I discuss the thermal dilepton rates, heavy quarkonium properties as well as the chiral and $U(1)_{A}$ symmetries at finite temperature.
\end{abstract}

\section{Introduction}

Understanding the phases and properties of strongly interacting matter at high temperature and density has been one of the central goals of research in nuclear science. The hadrons inside the medium would feel the modifications when the medium is heated or compressed [1]. The vector meson $\rho$ and the axial vector meson $a_{1}$ are degenerate when the chiral symmetry is restored from the spontaneously broken phase at a certain temperature. The particles $\eta^{\prime}$ and $\eta$ are degenerate when the $U(1)_{A}$ symmetry is restored. The heavy quarkonia, e.g. $J / \psi$ and $\Upsilon$, due to their small size and large mass, may survive up to certain critical temperatures and could serve as medium thermometers [2]. The thermal modifications of hadrons are encoded in the hadron spectral functions, e.g. the presence (absence) of a resonance peak in the spectral function indicates the existence (dissociation) of a bound state. The hadron spectral function is also related to thermal dilepton and photons rates as well as transport properties of the medium, e.g. the electrical conductivity and heavy quark diffusion coefficient. The related hadron correlation functions and their susceptibilities are useful observables that allow to study the chiral phase transition and the $U(1)_{A}$ symmetry restoration.

In the following I will give brief introductions to meson correlation and spectral functions in section 2 , and to the chiral and $U(1)_{A}$ symmetries in section 3. In section 4 I will show the recent lattice QCD results on in-medium hadron properties. For a review on earlier results see recent reviews, e.g. Ref. [3]. Finally I will give a summary in section 5.

\section{Meson correlators and spectral functions}

In-medium meson properties as well as some transport coefficients are all encoded in thermal meson spectral functions. The meson spectral function $\rho_{H}(\omega, \mathbf{p})$ for a given quantum number channel $H$ can be defined through the retarded correlator $D_{H}^{R}(\omega, \mathbf{p})[4]$

$$
\rho_{H}(\omega, \mathbf{p})=D_{H}^{+}(\omega, \mathbf{p})-D_{H}^{-}(\omega, \mathbf{p})=2 \operatorname{Im} D_{H}^{R}(\omega, \mathbf{p}),
$$

where

$D_{H}^{+(-)}(\omega, \mathbf{p})=\int \mathrm{d}^{4} x e^{i \omega t-i \mathbf{p x}} D_{H}^{+(-)}(t, \mathbf{x}), i D_{H}^{+}(t, \mathbf{x})=\left\langle J_{H}(t, \mathbf{x}) J_{H}(0, \mathbf{0})\right\rangle$ and $i D_{H}^{-}(t, \mathbf{x})=\left\langle J_{H}(0, \mathbf{0}) J_{H}(t, \mathbf{x})\right\rangle$. The local meson operator $J_{H}(t, \mathbf{x})$ has the form of $J_{H}(t, \mathbf{x})=\bar{\psi}(t, \mathbf{x}) \Gamma_{H} \psi(t, \mathbf{x})$, with $\Gamma_{H}=1, \gamma_{5}, \gamma_{\mu}, \gamma_{5} \gamma_{\mu}$, for scalar $(S C)$, pseudo scalar $(P S)$, vector $(V C)$ and axial vector $(A V)$ channels, respectively. The relation of these quantum numbers to different meson states is summarized in Table 1.

\footnotetext{
a e-mail: htding@quark.phy.bnl.gov
} 
Table 1. Meson states in different channels for light, charm and bottom quarks.

\begin{tabular}{|c|c|c|c|c|c|c|}
\hline Channel & $\Gamma_{H}$ & ${ }^{2 S+1} L_{J}$ & $J^{P C}$ & $u \bar{u}$ states & $c \bar{c}$ states & $b \bar{b}$ states \\
\hline PS & $\gamma_{5}$ & ${ }^{1} S_{0}$ & $0^{-+}$ & $\pi$ & $\eta_{c}$ & $\eta_{b}$ \\
VC & $\gamma_{\mu}$ & ${ }^{3} S_{1}$ & $1^{--}$ & $\rho$ & $J / \psi$ & $\gamma$ \\
SC & 1 & ${ }^{3} P_{0}$ & $0^{++}$ & $a_{0}$ & $\chi_{c 0}$ & $\chi_{b 0}$ \\
AV & $\gamma_{5} \gamma_{\mu}$ & ${ }^{3} P_{1}$ & $1^{++}$ & $a_{1}$ & $\chi_{c 1}$ & $\chi_{b 1}$ \\
\hline
\end{tabular}

The spectral function in the vector channel $\rho_{V}(\omega, \mathbf{p}, T)$ is related to the experimentally accessible differential cross section for thermal dilepton production,

$$
\frac{\mathrm{d} W}{\mathrm{~d} \omega \mathrm{d}^{3} \mathbf{p}}=C_{\mathrm{em}} \frac{\alpha_{e m}^{2}}{6 \pi^{3}} \frac{1}{\left(\omega^{2}-\mathbf{p}^{2}\right)\left(e^{\omega / T}-1\right)} \rho_{V}(\omega, \mathbf{p}, T),
$$

where $\alpha_{e m}$ is the electromagnetic fine structure constant and $C_{e m}$ is the sum of the square of the elementary charges of the quark flavor $f, C_{e m}=\sum_{f} Q_{f}^{2}$. The low frequency limit of the vector spectral function is related to the transport properties of the medium. In the light meson case, the spatial components of the vector spectral function yield the electrical conductivity

$$
\frac{\sigma}{T}=\frac{C_{e m}}{6} \lim _{\omega \rightarrow 0} \lim _{\mathbf{p} \rightarrow 0} \frac{\rho_{i i}(\omega, \mathbf{p}, T)}{\omega T},
$$

The electrical conductivity quantifies the response of the QGP to electromagnetic fields and is also related to the emission rate of soft photons [5]

$$
\lim _{\omega \rightarrow 0} \omega \frac{\mathrm{d} R_{\gamma}}{\mathrm{d}^{3} p}=\frac{3}{2 \pi^{2}} \sigma(T) T \alpha_{e m} .
$$

Furthermore, the photon emission rate of a thermal medium $\omega \frac{\mathrm{d} R_{\gamma}}{\mathrm{d}^{3} p}$ is also related to the light vector spectral function at light-like 4-momentum

$$
\omega \frac{\mathrm{d} R_{\gamma}}{\mathrm{d}^{3} p}=C_{e m} \frac{\alpha_{e m}}{4 \pi^{2}} \frac{\rho_{V}(\omega=|\mathbf{p}|, T)}{\mathrm{e}^{\omega / T}-1} .
$$

The spatial components of the heavy quark vector spectral function are related to the heavy quark diffusion coefficient $D$

$$
D=\frac{1}{6 \chi^{00}} \lim _{\omega \rightarrow 0} \lim _{\mathbf{p} \rightarrow 0} \sum_{i=1}^{3} \frac{\rho_{i i}^{V}(\omega, \mathbf{p}, T)}{\omega},
$$

where $\chi^{00}$ is the quark number susceptibility that is defined through the zeroth component of the temporal correlator in the vector channel. The heavy quark diffusion coefficient is also related to the ratio of shear viscosity over entropy density $\eta / s[6]$.

On the lattice, however, spectral functions cannot be computed directly. The quantity that can be directly computed is the Euclidean temporal correlation function $G_{H}(\tau, \mathbf{p})$. It is defined as

$$
G_{H}(\tau, \mathbf{p})=\int \mathrm{d}^{3} \mathbf{x} e^{-i \mathbf{p} \cdot \mathbf{x}}\left\langle J_{H}(\tau, \mathbf{x}) J_{H}(0, \mathbf{0})\right\rangle,
$$

where $G_{H}(\tau, \mathbf{p})$ is the analytic continuation of $D^{+}(t, \mathbf{p})$ from real to imaginary time $G_{H}(\tau, \mathbf{p})=D^{+}(-i \tau, \mathbf{p})$. By using the KMS relation and the above equation, one can relate the correlation function to the spectral function,

$$
G_{H}(\tau, \mathbf{p})=\int_{0}^{\infty} \frac{\mathrm{d} \omega}{2 \pi} \rho_{H}(\omega, \mathbf{p}, T) \frac{\cosh (\omega(\tau-1 / 2 T))}{\sinh (\omega / 2 T)} .
$$

The above equation is the basic relation for extracting spectral functions from meson correlation functions calculated in the imaginary time formalism. To extract the spectral function reliably one needs 
large number of points in the temporal direction of the lattice. Normally the Maximum Entropy Method (MEM) is used to extract spectral functions from correlators [7]. As the temporal extent, $a N_{\tau}$, is restricted by the inverse temperature, in order to have larger number of points $N_{\tau}$ in the temporal direction one has to decrease lattice spacing $a$ at fixed values of temperature. As a consequence the computing cost will increase. The Euclidean spatial correlation function $G_{H}\left(z, \mathbf{p}_{\perp}, p_{\tau}\right)$ can be computed via an integration over the Euclidean time $\tau$ and two spatial directions $\mathbf{x}_{\perp}=(x, y)$

$$
G_{H}\left(z, \mathbf{p}_{\perp}, p_{\tau}\right)=\int_{0}^{1 / T} \mathrm{~d} \tau \int \mathrm{d} \mathbf{x}_{\perp} \exp (-i \tilde{\mathbf{p}} \cdot \tilde{\mathbf{x}})\left\langle J_{H}(\tau, \mathbf{x}) J_{H}(0, \mathbf{0})\right\rangle,
$$

where $\mathbf{p}_{\perp}=\left(p_{x}, p_{y}\right), \tilde{\mathbf{x}}=\left(\mathbf{x}_{\perp}, \tau\right)$ and $\tilde{\mathbf{p}}=\left(\mathbf{p}_{\perp}, p_{\tau}\right)$. The spatial correlation function is related to the spectral function through

$$
G_{H}\left(z, \mathbf{p}_{\perp}, p_{\tau}\right)=\int_{-\infty}^{\infty} \frac{\mathrm{d} p_{z}}{2 \pi} \exp \left(i p_{z} z\right) \int_{0}^{\infty} \frac{\mathrm{d} \omega}{2 \pi} \frac{2 \omega}{\omega^{2}+p_{\tau}^{2}} \rho_{H}\left(\omega, \mathbf{p}_{\perp}, p_{z}, p_{\tau}, T\right) .
$$

The above equation is much more complicated than that of the temporal correlation function and it thus is not so straightforward to extract the spectral function from the spatial correlation function. However, one advantage of spatial correlation functions over temporal correlation functions is that the physical extent in the spatial direction is not restricted by the temperature and thus by going to large distances one can extract the exponentially decayed constant, i.e. screening mass.

Bottomonium states are too heavy to be implemented in direct lattice QCD calculations. The lattice spacing required for this would need to be much smaller than the inverse of the bottom quark mass. A solution is provided by the Non-Relativistic QCD (NRQCD), which is an effective theory of QCD where physics above the scale of the heavy quark mass $M$ is integrated out. In the framework of NRQCD, it is sufficient to have the lattice spacing $a$ as coarse as $M \gg 1 / a \gg M v$. Thus by construction there is no continuum limit of NRQCD on the lattice. The correlation function in NRQCD is related to the spectral function through [8]

$$
G_{H}(\tau, \mathbf{p})=\int_{0}^{\infty} \frac{\mathrm{d} \omega}{2 \pi} \rho_{H}(\omega, \mathbf{p}, T) \exp (-\omega \tau) \quad \text { (NRQCD). }
$$

Note that the integral kernel $\exp (-\omega \tau)$ has no temperature dependence and the correlation function $G_{H}(\tau, \mathbf{p})$ in Eq. (11) is not symmetric at $\tau=1 / 2 T$. These are in contrast to $G_{H}(\tau, \mathbf{p})$ defined in Eq. (8).

\section{Chiral and axial symmetries}

The QCD Lagrangian with $N_{f}$ massless quark flavors has a large group of symmetry

$$
S U\left(N_{f}\right)_{L} \otimes S U\left(N_{f}\right)_{R} \otimes U(1)_{V} \otimes U(1)_{A} .
$$

In the vacuum the chiral symmetry $S U\left(N_{f}\right)_{L} \otimes S U\left(N_{f}\right)_{R}$ of QCD is spontaneously broken into a smaller $S U\left(N_{f}\right)_{V}$ vacuum symmetry. It is expected that at sufficiently high temperature the QCD matter will transit from a chiral asymmetric phase to_a chiral restored phase. The order parameter for such a phase transition is the chiral condensate $\langle\bar{\psi} \psi\rangle$, which is nonzero below the chiral phase transition temperature $T_{c}$ and zero above $T_{c}$. The chiral symmetry restoration can also be signaled by the degeneracy of the vector and axial vector mesons, e.g. $\rho$ and $a_{1}$.

The breaking of chiral symmetry $S U(3)_{L} \otimes S U(3)_{R}$ predicts the existence of nine massless Goldstone bosons. However, there are only eight light mesons in nature, i.e. octet mesons $\pi^{ \pm}, \pi^{0}, K^{ \pm}, K^{0}$, $\overline{K^{0}}, \eta$. The ninth "Goldstone" boson, i.e. the singlet meson $\eta^{\prime}(958)$, has a much larger mass. Such a mass splitting is due to the violation of the axial $U(1)_{A}$ symmetry by the axial anomaly present at the quantum level $[9,10]$. The effective restoration of the $U(1)_{A}$ symmetry may lead to a softening of the $\eta^{\prime}$ mass which would provide interesting experimental signatures.

The degree of $U(1)_{A}$ symmetry breaking may affect our understanding of QCD phase diagram. In the chiral limit of two flavor QCD, the order of the chiral phase transition is expected to be second 


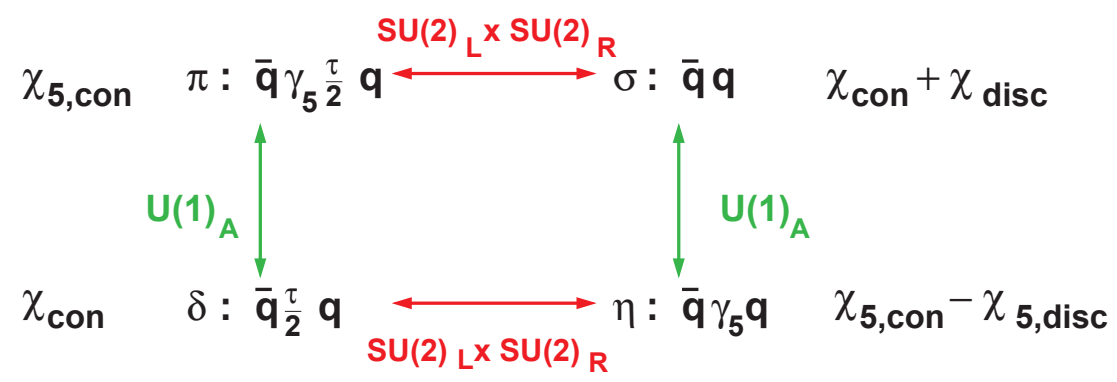

Fig. 1. Symmetry transformations relating pseudo scalar and scalar mesons in flavor non-singlet and singlet channels in $N_{f}=2$ QCD [11].

order belonging to the three dimensional $\mathrm{O}(4)$ universality class if the $U(1)_{A}$ is strongly broken near the phase transition. If the $U(1)_{A}$ symmetry breaking is negligible or $U(1)_{A}$ is restored near the phase transition then the chiral phase transition can become first order [12]. Thus the nature of the chiral phase transition may depend critically on the strength of $U(1)_{A}$ symmetry breaking.

The way to investigate the chiral and $U(1)_{A}$ symmetry on the lattice is to look at various correlation functions and susceptibilities. The susceptibilities $\chi_{\pi, \delta, \sigma, \eta}$ are the four volume integration of the corresponding correlation functions. $\chi_{\text {disc }}$ is the disconnected part of the susceptibility of the chiral condensate $\langle\psi \psi \psi\rangle$, i.e. $\chi_{\text {disc }}=\left\langle(\bar{\psi} \psi)^{2}\right\rangle-\langle\psi \psi \psi\rangle^{2}$ and it equals to the disconnected part of $\chi_{\sigma} \cdot \chi_{5 \text {, disc }}$ is the disconnected part of the susceptibility of $\left\langle\bar{\psi} \gamma_{5} \psi\right\rangle$, i.e. $\chi_{5 \text {, disc }}=\left\langle\left(\bar{\psi} \gamma_{5} \psi\right)^{2}\right\rangle-\left\langle\left(\bar{\psi} \gamma_{5} \psi\right)\right\rangle^{2}$ and it equals to disconnected part of $\chi_{\eta}$. They can also be written in terms of other susceptibilities as shown in Fig. 1 and thus the $U(1)_{A}$ and chiral symmetries are restored through the following degeneracies:

$$
\begin{aligned}
U(1)_{A} \text { symmetry: } & \chi_{\pi}=\chi_{\delta} \text { and } \chi_{\delta}+\chi_{\text {disc }}=\chi_{\pi}-\chi_{5, \text { disc }}, \\
S U(2)_{L} \times S U(2)_{R} \text { symmetry: } & \chi_{\pi}=\chi_{\delta}+\chi_{\text {disc }} \text { and } \chi_{\delta}=\chi_{\pi}-\chi_{5, \text { disc }} .
\end{aligned}
$$

We see that if $U(1)_{A}$ symmetry is restored the values of $\chi_{\pi}-\chi_{\delta}$ and $\chi_{\text {disc }}+\chi_{5 \text {, disc }}$ should both go to zero and if chiral symmetry is restored $\chi_{\pi}-\chi_{\delta}$ should equal to $\chi_{\text {disc }}$ and $\chi_{\text {disc }}-\chi_{5 \text {,disc }}$ should be zero. When both chiral and $U(1)_{A}$ symmetries are restored, $\chi_{\text {disc }}$ and $\chi_{5, \text { disc }}$ should both equal to zero.

\section{Lattice QCD results}

\subsection{Thermal dilepton rates, electrical conductivity and soft photon emission rate}

Recently a detailed calculation of the light vector correlation function on large isotropic quenched lattice was reported at a fixed value of temperature $T \simeq 1.45 T_{c}$ [5]. Calculations have been performed at several lattice spacings and volumes. This enables a reliable extrapolation of the vector correlation function to the continuum limit for a large Euclidean time interval $0.2 \leq \tau T \leq 0.5$. From the comparison of the free correlation function and the continuum extrapolated vector correlation function at $T \simeq 1.45 T_{c}$, it was found that the high energy part of the spectral function at $T \simeq 1.45 T_{c}$ is quite close to the free field theory, thus the following ansatz for the spectral function is used to analyze the continuum extrapolated vector correlation function

$$
\rho_{i i}(\omega)=2 \chi_{q} c_{B W} \frac{\omega \Gamma / 2}{\omega^{2}+(\Gamma / 2)^{2}}+\frac{3}{2 \pi}(1+k) \omega^{2} \tanh (\omega / 4 T) \Theta\left(\omega_{0}, \Delta_{\omega}\right),
$$

where $c_{B W}$ and $\Gamma$ are fit parameters to adjust the Breit-Wigner (BW) term, and $k$ is a fit parameter accounting for corrections to the free field theory in the second term. To understand the systematic errors that arise from a specific fit ansatz, a function $\Theta\left(\omega_{0}, \Delta_{\omega}\right)=\left(1+\mathrm{e}^{\left(\omega_{0}^{2}-\omega^{2}\right) / \omega \Delta_{\omega}}\right)^{-1}$ is also adopted in the second term of Eq. (15). In the limit of $\Delta_{\omega} \rightarrow 0$ the function $\Theta\left(\omega_{0}, \Delta_{\omega}\right)$ becomes a step function with discontinuity at $\omega_{0}$. Fits with two extreme cases of parameter sets of $\omega_{0}$ and $\Delta_{\omega}$ that are constrained 
by data are shown in Fig. 2. In the left panel of Fig. 2 the thermal dilepton rates are compared to those calculated within the hard thermal loop (HTL) approximation [13] using a thermal quark mass $m_{T} / T=1$. Obviously they are in good agreement for $\omega / T \gtrsim 2$. For $1 \lesssim \omega / T \lesssim 2$ differences between the HTL spectral function and lattice results is about a factor of two, which also is the intrinsic uncertainty in the spectral analysis. At energies $\omega / T \lesssim 1$ the HTL results grow too rapidly [13], as is well known. As the results shown in Fig. 2 is only for one single temperature, it is important to have results from lattice $\mathrm{QCD}$ at various temperatures around $T_{c}$ in the near future.
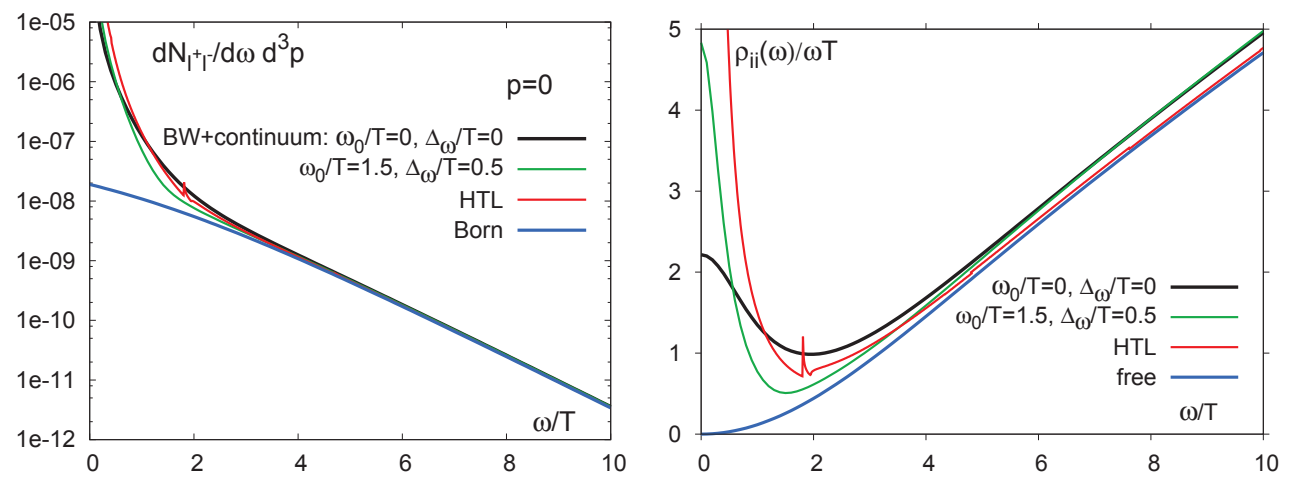

Fig. 2. Left: thermal dilepton rate in 2 -flavor QCD. Shown are results from fits using Eq. (15) without a cut-off on the continuum contribution $\left(\omega_{0} / T=0\right)$ and with the largest cut-off tolerable in the fit ansatz $\left(\omega_{0} / T=1.5\right)$. The HTL curve is for a thermal quark mass $m_{T} / T=1$ and the Born rate is obtained by using the free spectral function. Right: the spectral functions that entered the calculation of the dilepton rate [5].

The vector spectral function is shown in the right panel of Fig. 2. There is no resonance peak seen in the spectral function. Through Eq. (3) the slope of the spectral function at vanishing energy gives an estimate for the electrical conductivity and the zero energy limit of the thermal photon rate [5]

$$
1 / 3 \lesssim \frac{1}{C_{e m}} \frac{\sigma}{T} \lesssim 1, \quad \lim _{\omega \rightarrow 0} \omega \frac{\mathrm{d} R_{\gamma}}{\mathrm{d}^{3} p}=(0.0004-0.0013) T_{c}^{2} \quad \text { at } \quad T \simeq 1.45 T_{c} .
$$

Different values of the electrical conductivity from lattice QCD calculations were reported in Ref. [14, 15], however, these values, unlike those reported in Ref. [5], are unrenormalized and not extrapolated to the continuum limit. The temperature dependence of the electrical conductivity has also been studied on the lattice recently. It was found that $\sigma / T \approx 0.33 C_{e m}$ and is almost independent on temperature in the temperature range $1.16 T_{c} \lesssim T \lesssim 2.98 T_{c}$ [16] . However, the results reported in Ref. [16] were obtained at only one value of the lattice cutoff and were not extrapolated to the continuum limit. Nevertheless these results are consistent with those in Ref. [5]. The leading order pQCD calculations give a much larger result, i.e. $\sigma / T \approx 6 e^{2}$ [17]. Note also that the electrical conductivity of a pion gas decreases with temperature [18] while in the deconfined phase it increases with $T$ [16].

\subsection{Heavy quarkonia correlators and spectral functions}

The most direct approach to check whether a quarkonium state survives in the hot medium is to investigate the temperature dependence of its spectral function. Recently a detailed analysis of the charmonium temporal correlation and spectral functions computed on fine and large isotropic quenched lattices at $T=0.73,1.46,2.20$ and $2.93 T_{c}$ was reported in Ref. [19]. Computing time can be saved by doing simulations on anisotropic lattice but the lattice cutoff effects are much more severe in this case as discussed in Ref. [19]. This work is mainly based on the MEM to extract the spectral functions. As the number of data points in the temporal correlation function is very important in this type of study, an enormous computing effort has been made to have large isotropic lattices, e.g. $128^{3} \times 48$ at 
$1.46 T_{c}$. The uncertainties on the spectral function from MEM have been carefully studied. The results for the charmonium spectral function in the vector channel is shown in the left panel of Fig. 3. It is obvious that at $1.46 T_{c}$ the ground state peak seen at $0.73 T_{c}$ disappears, i.e. $J / \psi$ does not survive at $T \geqslant 1.46 T_{c} . \eta_{c}, \chi_{c 0}$ and $\chi_{c 1}$ are also found to disappear at $T \geqslant 1.46 T_{c}$ [19]. The fate of $\mathrm{S}$ wave sates $\left(J / \psi\right.$ and $\left.\eta_{c}\right)$ suggested by the analysis in Ref. [19] is different from previous lattice QCD studies that suggested the survival of $\mathrm{S}$ wave sates up to $2 T_{c}$. This is mainly due to the fact that most of the previous lattices studies were done on anisotropic lattices and had a fewer number of points in the temporal direction [19]. On the other hand, the results for P wave states $\left(\chi_{c 0}\right.$ and $\left.\chi_{c 1}\right)$ from this work are compatible with previous results, i.e. they does not survive at $T \geqslant 1.46 T_{c}$. However, these results from Ref. [19] do not necessarily mean that all the charmonium states melt at the same temperature. One needs further lattice QCD computations at $T_{c}<T<1.46 T_{c}$ to locate the dissociation temperatures of charmonium states.
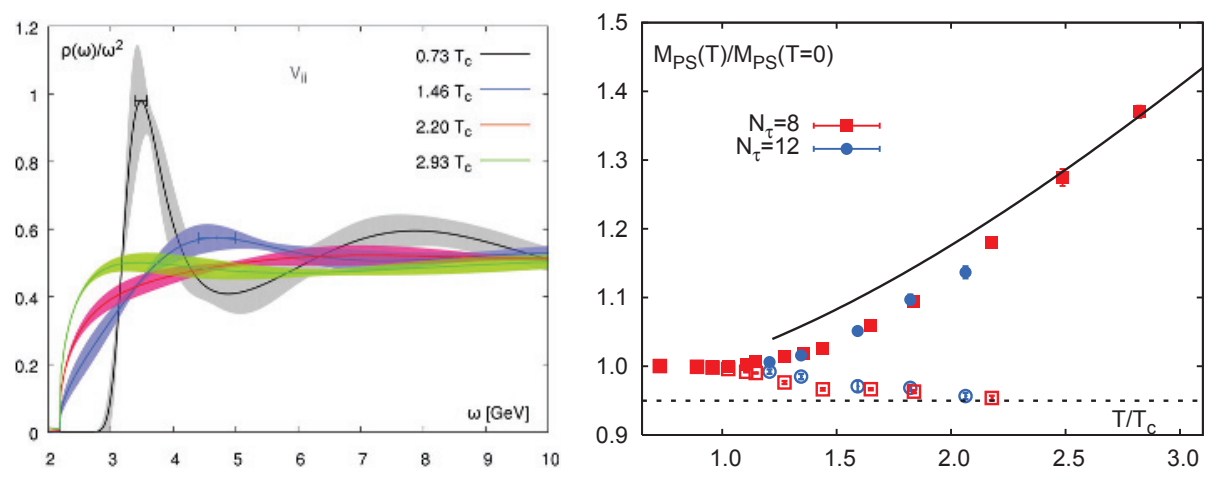

Fig. 3. Left: Statistical uncertainties of output spectral functions in the $V_{i i}$ channel at temperatures $T=$ $0.73,1.46,2.20,2.93 T_{c}$. The shaded areas are statistical errors of amplitudes of output spectral functions from Jackknife analyses and the solid lines inside the shaded areas are mean values of spectral functions. The horizontal error bars at the first peaks of spectral functions at $0.73 T_{c}$ and $1.46 T_{c}$ stand for the statistical uncertainties of the peak location obtained from Jackknife analyses [19]. Right: Pseudo scalar charmonium screening masses calculated with antiperiodic (filled symbols) and periodic (open symbols) boundary conditions as function of the temperature. Solid (dashed) lines correspond to the free theory prediction with charm quark mass $m_{c}=1.42 \mathrm{GeV}$ for antiperiodic (periodic) boundary conditions [20].

Spatial charmonium correlators and screening masses have also been calculated on the lattice in full QCD [20]. As shown in the right panel of Fig. 3, at $T \gtrsim 1.5 T_{c}$ the screening mass in the pseudo scalar channel differs strongly from its zero temperature value and it depends strongly on the boundary conditions. All this suggests the dissociation of bound states in the pseudo scalar channel at $T \gtrsim 1.5 T_{c}$. This finding is consistent with that from the study of charmonium spectral functions extracted from temporal correlation functions reported in Ref. [19].

For the fate of bottomonia at finite temperature, a lattice calculation in the framework of NRQCD has been performed recently [8]. Lattice QCD simulations were performed with two light quarks on highly anisotropic lattice using NRQCD for bottom quarks. The spectral functions shown in Fig. 4.2 are extracted from correlations functions using the MEM. It can clearly be seen from that figure that the ground state in the $\gamma$ channel survives up to $2.09 T_{c}$. Results for the $\eta_{b}$ channel are similar. The calculation has also been extrapolated exploringly to nonzero baryon density [21]. However, one has to note that there is no continuum limit for the lattice version of NRQCD and the calculations in Ref. [8, 21 were performed on highly anisotropic lattices that can distort the physics.

The properties of heavy quarkonia in the vacuum have been studied based on a non relativistic Schrödinger equation using either modeled or lattice QCD computed heavy quark potentials. However, the definition of the heavy quark potential (free energy or internal energy ) is ambiguous at nonzero 


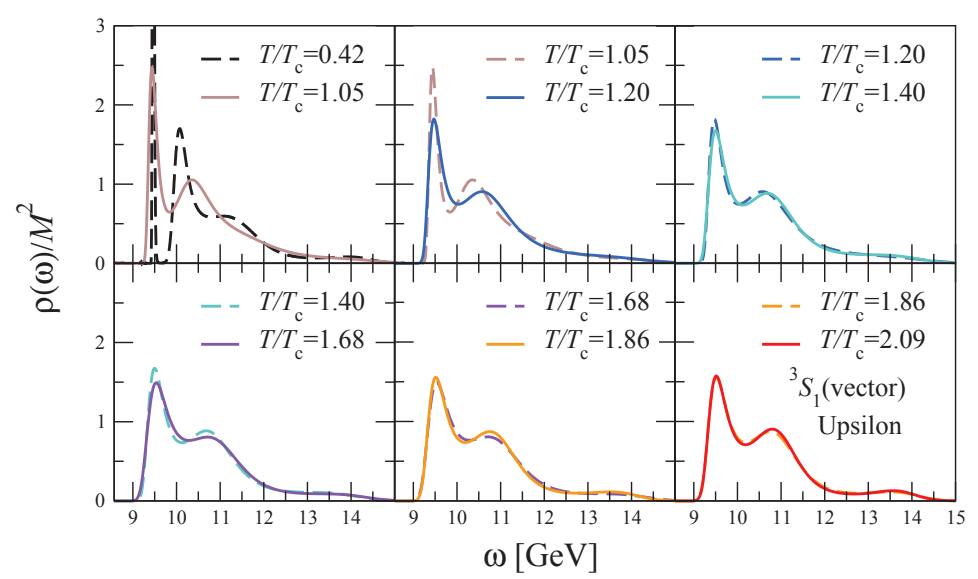

Fig. 4. Bottomonium spectral functions in the vector channel from Non-Relativisitic QCD calculations [8].

temperature. Recently it has been proposed by authors in Ref. [22] that in the nonrelativistic limit the leading order potential in an effective Schrödinger equation can be appropriately defined by assuming that the spectral density of the Wilson loop has peaks according to Breit-Wigner distributions. In this framework, the peak location of the spectral density corresponds to the real part of the heavy quark potential while the peak width corresponds to the imaginary part of the heavy quark potential. Using the MEM it is feasible to extract the peak location, however, it is very hard to extract the width of the peak reliably.

The charm diffusion coefficient obtained from the slope of the vector spectral function is almost $1 / \pi T$ at $T \in\left(1.46 T_{c}, 2.93 T_{c}\right)$ and compatible with zero below $T_{c}$ [19]. The mean value of the heavy quark diffusion coefficient obtained from the computation of heavy quark effective theory on the lattice is somewhat larger $[23,24]$, i.e. $2 / \pi T$ but compatible with the charm diffusion coefficient from Ref. [19] within errors. These values from lattice QCD are smaller than that from pQCD calculations in the investigated temperature window [25] and lies in the range used in phenomenological models [26] as well as non-perturbative models [27].

\subsection{Chiral phase transition and $U(1)_{A}$ symmetry restoration}

Studies on this topic have also been carried out recently in Ref. [28]. In this study the improved (p4) staggered action was used and the effective restoration of the $U(1)_{A}$ symmetry occurs at a temperature larger than the QCD transition temperature indicated from the degeneracy of the screening masses of pseudo scalar and scalar correlators. A better discretized version of staggered fermions has also been implemented in Ref. [29] to investigate the fate of the axial symmetry at finite temperature and their results suggests that $U(1)_{A}$ symmetry still remains broken at about $1.1 T_{c}$. Due to subtle issues concerning the realization of chiral and axial symmetries in the staggered discretized fermion action, better discretization schemes, e.g. domain wall fermions and overlap fermions, which preserve the full chiral symmetry of continuum QCD and reproduce the correct axial anomaly even at nonzero lattice spacing, have also been implemented to study this topic.

Using domain wall fermions the HotQCD collaboration recently investigated the restoration of the spontaneously broken chiral symmetry and the effective restoration of the anomalously broken $U(1)_{A}$ symmetry at finite temperature on $16^{3} \times 8$ lattices with $m_{\pi} \approx 200 \mathrm{MeV}$ [11]. One of the main results is shown in Fig. 4.3. The three quantities, $\chi_{\text {disc }}, \chi_{5 \text {,disc }}$ and $\chi_{\pi}-\chi_{\delta}$, agree within errors for temperature $T \gtrsim 170 \mathrm{MeV}$. This suggests the restoration of the $S U(2)_{L} \times S U(2)_{R}$ symmetry according to Eq. (14). The $U(1)_{A}$ symmetry on the other hand, remains broken up to temperature $T \approx 200 \mathrm{MeV}$ as indicated from the nonzero value of $\chi_{\pi}-\chi_{\delta}$. If $170 \mathrm{MeV}$ is taken as the pseudo critical transition temperature, this indicates that $U(1)_{A}$ still remains broken at $T \approx 1.2 T_{p c}$. As shown in the right panel 

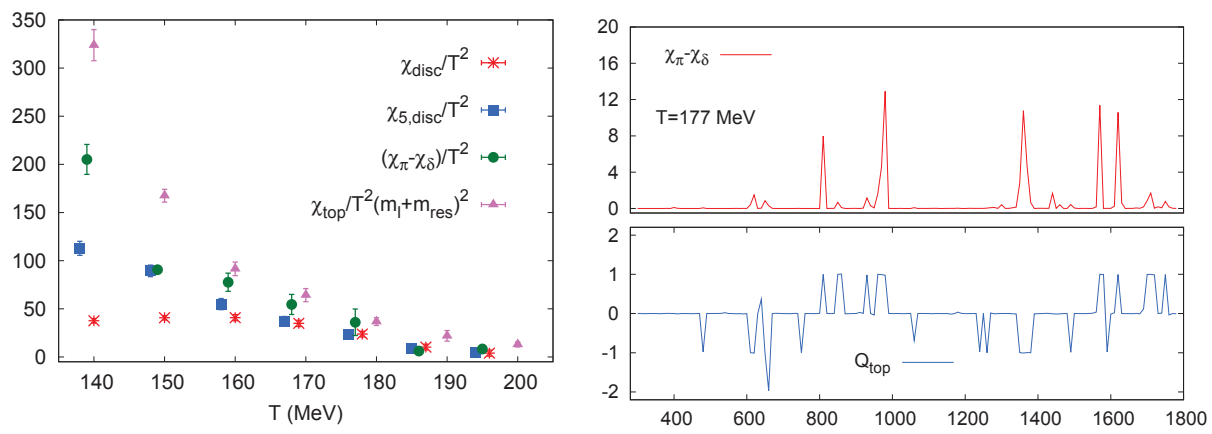

Fig. 5. Left: susceptibilities as function of temperature, Right: the time histories of the topological charge $Q_{t o p}$ and $\chi_{\pi}-\chi_{\delta}$. Figures are taken from Ref. [11].

of Fig. 4.3, the nonzero value of $\chi_{\pi}-\chi_{\delta}$ is related to the nonzero topological charge $Q_{t o p}$, which will be suppressed in the large volume limit. To draw reliable conclusion on $U(1)_{A}$ symmetry restoration at high temperature, one needs to carry out simulations with larger volumes and perform detailed analysis on the Dirac operator eigenvalue spectrum. The JLQCD collaboration using overlap fermions performed simulations at higher temperatures mainly on $16^{3} \times 8$ lattices with $m_{\pi} \gtrsim 290 \mathrm{MeV}$ and $Q_{\text {top }}$ fixed to be zero [30]. They found that $U(1)_{A}$ symmetry is restored at $209 \mathrm{MeV}$ by looking at the degeneracies of correlators and the gap around origin in the Dirac operator eigenvalue spectrum. However, the volume effects on topological charge fixing might be large and need to be checked.

From the studies discussed above, the effective $U(1)_{A}$ symmetry restoration seems to occur after the chiral symmetry restoration and $U(1)_{A}$ restoration temperature certainly needs further studies. Of course, it would be very interesting to have good signals to probe the possible restoration of $U(1)_{A}$ symmetry in the heavy ion experiments [31-33].

\section{Summary}

The lattice QCD calculations presented here show that there has been significant progress in our understanding of strongly interacting matter through the in-medium hadron properties in recent years. However, more needs to be done on the lattice to help to understand the heavy ion experiment data. The $U(1)_{A}$ symmetry restoration that is being intensively studied on the lattice may be observed in the heavy ion experiments from some interesting signals, e.g. a softening of the $\eta^{\prime}$ mass.

\section{Acknowledgements}

The work is supported under contract No. DE-AC02-98CH10886 with the U.S. Department of Energy. HTD thanks Prasad Hegde and Swagato Mukherjee for useful discussions and Frithjof Karsch for critical reading of the manuscript.

\section{References}

1. R. Rapp, B. Kampfer, A. Andronic, D. Blaschke, et al., Lect. Notes Phys. 814 (2011) 335.

2. T. Matsui and H. Satz, Phys. Lett. B 178 (1986) 416.

3. C. DeTar and U. M. Heller, Eur. Phys. J. A 41 (2009) 405 [arXiv:0905.2949 [hep-lat]].

4. J. -P. Blaizot and E. Iancu, Phys. Rept. 359 (2002) 355.

5. H. -T. Ding, A. Francis, O. Kaczmarek, et al., Phys. Rev. D 83 (2011) 034504.

6. R. Rapp and H. van Hees, arXiv:0803.0901 [hep-ph]. 
7. M. Asakawa, T. Hatsuda and Y. Nakahara, Prog. Part. Nucl. Phys. 46 (2001) 459 [hep-lat/0011040].

8. G. Aarts et al., JHEP 1111 (2011) 103, Phys. Rev. Lett. 106 (2011) 061602.

9. S. L. Adler, Phys. Rev. 177 (1969) 2426.

10. J. S. Bell and R. Jackiw, Nuovo Cim. A 60 (1969) 47.

11. A. Bazavov, et al. [HotQCD Collaboration], arXiv:1205.3535 [hep-lat].

12. R. D. Pisarski and F. Wilczek, Phys. Rev. D 29 (1984) 338.

13. E. Braaten, R. D. Pisarski, T. -C. Yuan, Phys. Rev. Lett. 64, 2242 (1990).

14. S. Gupta, Phys. Lett. B 597 (2004) 57.

15. G. Aarts, C. Allton, J. Foley, S. Hands and S. Kim, Phys. Rev. Lett. 99 (2007) 022002.

16. A. Francis and O. Kaczmarek, Prog. Part. Nucl. Phys. 67 (2012) 212 [arXiv:1112.4802 [hep-lat]].

17. P. B. Arnold, G. D. Moore and L. G. Yaffe, JHEP 0305 (2003) 051.

18. D. Fernandez-Fraile and A. Gomez Nicola, Eur. Phys. J. C 62 (2009) 37.

19. H. -T. Ding, A. Francis, O. Kaczmarek, et al., arXiv:1204.4945 [hep-lat].

20. F. Karsch, E. Laermann, S. Mukherjee and P. Petreczky, Phys. Rev. D 85 (2012) 114501.

21. S. Hands, S. Kim and J. -I. Skullerud, Phys. Lett. B 711 (2012) 199 [arXiv:1202.4353 [hep-lat]].

22. A. Rothkopf, T. Hatsuda and S. Sasaki, Phys. Rev. Lett. 108 (2012) 162001.

23. A. Francis, O. Kaczmarek, M. Laine and J. Langelage, PoS LATTICE 2011 (2011) 202.

24. D. Banerjee, S. Datta, R. Gavai and P. Majumdar, Phys. Rev. D 85 (2012) 014510.

25. S. Caron-Huot and G. D. Moore, Phys. Rev. Lett. 100 (2008) 052301 [arXiv:0708.4232 [hep-ph]].

26. P. Gossiaux, J. Aichelin and T. Gousset, arXiv:1201.4038 [hep-ph].

27. M. He, R. J. Fries and R. Rapp, arXiv:1204.4442 [nucl-th].

28. M. Cheng et al., Eur. Phys. J. C 71 (2011) 1564 [arXiv:1010.1216 [hep-lat]].

29. H. Ohno, U. M. Heller, F. Karsch and S. Mukherjee, PoS LATTICE 2011 (2011) 210.

30. G. Cossu, et al., PoS LATTICE 2011 (2011) 188 [arXiv:1204.4519 [hep-lat]].

31. Z. Huang and X. -N. Wang, Phys. Rev. D 53 (1996) 5034.

32. J. I. Kapusta, D. Kharzeev and L. D. McLerran, Phys. Rev. D 53 (1996) 5028.

33. T. Csorgo, R. Vertesi and J. Sziklai, Phys. Rev. Lett. 105 (2010) 182301. 